\title{
Determining the pattern of whole genome methylation in patients with juvenile myoclonic epilepsy
}

\author{
Beatriz Lopes*, Danielle Bruno, Marina Gonsales, Marina Alvim, Clarissa Yasuda, Fernando Cendes, Iscia \\ Lopes-Cendes
}

\begin{abstract}
According to the World Health Organization, there are more than 50 million individuals with epilepsy worldwide; approximately $10 \%$ of these patients have juvenile myoclonic epilepsy (JME). JME is considered one of the most common forms of the genetic generalized epilepsies. Currently, it is believed that most patients with JME have a complex inheritance with different genetic and non-genetic factors involved. Therefore, we hypothesize that there may be epigenetic factors that influence the pattern of DNA methylation, which could be involved in the predisposition of JME. To test our hypothesis, we will analyze the pattern of DNA methylation in patients with JME and compared it to control individuals. We will use the whole-genome bisulfite sequencing (WGBS) technique. This is the first study accessing differentially methylated regions throughout the genome in JME patients. Our results may lead to a better understanding of the different etiological factors which may be predisposing to JME.
\end{abstract}

\section{Keywords:}

Juvenile Myoclonic Epilepsy, Epigenetic, methylation pattern

\section{Introduction}

Juvenile Myoclonic Epilepsy affects more than 5 million people worldwide $[1,2]$. The disease is characterized by the presence of myoclonic jerks with or without other types of generalized seizures. JME is the most common of the genetic generalized epilepsies (GGEs) [3,4]. Current evidence points to a complex inheritance in most GGEs, and although many candidate genes have been identified, most patients with JME do not have mutations identified. Thus, environmental factors may be influencing the predisposition to seizures in patients with JME [6]. Epigenetic mechanisms are affected by environmental factors, modifying the genic expression without changing the DNA sequence. Which leads to our hypothesis that epigenetic modifications, such as differential DNA methylation, may be involved in the predisposition to JME.

\section{Results and Discussion}

We extracted DNA from the peripheral blood of three patients with JME, and three healthy controls. The patients are followed at the University Hospital $(\mathrm{HC})$ at UNICAMP according to a detailed research protocol, including clinical and neuroimaging evaluations.

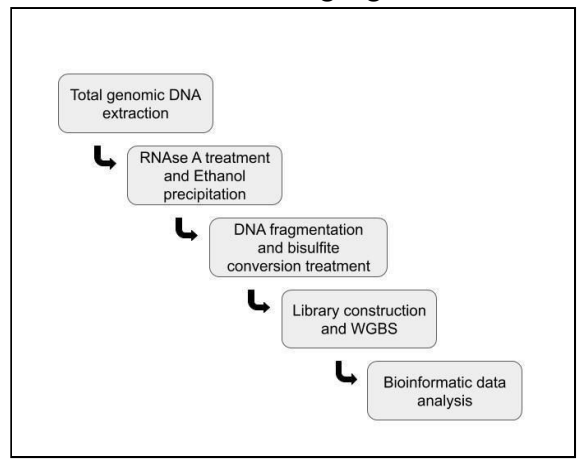

Figure 1. Method workflow

We are standardizing our steps; a good sample treatment will result in reliable data for further analysis. Wherefore, we used a test sample to determine and standardize these steps. We have found that treating our samples with RNAse $A$ and precipitating them with ethanol leads to the best results in the DNA preparation step. We have also tested the quality parameters of each patient and control DNAs by. visualizing DNA integrity in $0.8 \%$ agarose gels and using the Epoch ${ }^{\circledR}$ method. On average, the concentration of our samples is $200 \mathrm{ng} / \mu \mathrm{L}$ and purity of 1.7 with 0.15 of standard deviation. We expect to have the samples sequenced (WGBS) within the next month.

\section{Conclusions}

In conclusion, we established the best method of treatment and the bisulfite conversion procedures for our DNA samples.

This is the first study seeking to analyze the methylation pattern in the whole genome of patients with JME. Therefore, we expect that our results may help to better understand the role of epigenetic factors in the predisposition to JME.

\section{Acknowledgment}

We would like to thank the Department of Medical Genetics and Genomic Medicine and the Department of Neurology both located at the School of Medical Sciences at UNICAMP, and also the Brazilian Institute of Neuroscience and Neurotechnology (BRAINN), as well FAPESP for supporting this study.

[1] Fisher, R. S. et al., doi: 10.1111/j.0013-9580.2005.66104.x, [2] Robert S Fisher et al., doi: 10.1111/epi.12550, [3] Mirian S.B. Guaranha et al., doi: https://doi.org/10.1016/j.seizure.2010.10.004., [4] Jallon, P. et al., doi:10.1111/j.1528-1167.2005.00309.x, [5] Santos BPD et al., doi: 10.1371/journal.pone.0179629. eCollection 2017. [6] Chen $\mathrm{T}$ et al., doi:10.2147/NDT.S142032 